\title{
Hierarchical Strategy of Model Partitioning for VLSI-Design Using an Improved Mixture of Experts Approach
}

\author{
K. Hering \& R. Haupt \\ Universität Leipzig, Inst. für Informatik \\ Augustusplatz 10/11 \\ 04109 Leipzig, Germany
}

\author{
Th. Villmann \\ Inst. für Techno- und Wirtschaftsmathematik \\ E.-Schrödinger-Str., Geb. 48/575 \\ 67663 Kaiserslautern, Germany
}

to appear in the Proceedings of the 10th Workshop on Parallel and Distributed Simulation (PADS'96)

KEYwords. Partitioning, VLSI-Design, Logic Simulation, Genetic Algorithms, Hierarchical Mixture of Expers Strategy

\begin{abstract}
The partitioning of complex processor models on the gate and register-transfer level for parallel functional simulation based on the clock-cycle algorithm is considered. We introduce a hierarchical partitioning scheme combining various partitioning algorithms in the frame of a competing strategy. Melting together different partitioning results within one level using superpositions we crossover to a mixture of experts one. This approach is improved applying genetic algorithms. In addition we present two new partitioning algorithms both of them taking cones as fundamental units for building partitions.
\end{abstract}

\section{Introduction}

Logic design for whole microprocessor structures is accompanied with time-extensive simulation processes. Within the design strategy outlined in [14] the verification of functional (logical) behavior is strictly separated from the analysis of timing aspects. In this context the background of the present paper is given by simulation processes for functional design verification on gate and register-transfer level (logic simulation) where sequences of machine instructions or microcode are taken as test cases and underlying models comprise complex parts of processor structures. Under these assumptions the usage of cycle-based simulators is to be preferred. TEXSIM ${ }^{1}$ is a high performance simulator for logic simulation of synchronous designs

\footnotetext{
${ }^{1}$ copyright by IBM
}

using the clock-cycle algorithm. To achieve a significant reduction of running time for simulations the task is to parallelize them. Thereby a parallel TEXSIM simulation consists of several co-operating TEXSIM instances running on loosely coupled RS/6000 processors (system SP2 of IBM) over parts of the whole model. As a basic assumption, the process of the evaluation of combinational logic during the parallel simulation of a cycle has to be left unchanged. Therefore special fan-in cones are chosen as building blocks for model partitioning. A partition is directly related to certain workloads of the processors involved in later parallel simulation and communication overhead between co-operating TEXSIM instances and, hence, to the speed-up possible due to parallelization. The amount of time acceptable for partitioning depends on the expected total duration of all simulation runs to be performed regarding to a corresponding model. Simulation processes we are dealing with are characterized by a large number of time-extensive runs concerning a given model.

\section{Definitions}

First, we define a structural model for the logic design on gate and register-transfer level. The underlying hardware is supposed to be synchronous. Basic components are given by the sets $M_{I}, M_{O}, M_{E}, M_{L}, M_{S}$ (global inputs, global outputs, logical elements, storing elements, signals). $M_{E}$ includes all elements which represent combinational logic within the hardware to be simulated. Signals of $M_{S}$ are interpreted as wires. The elements of the set $M_{L}$ possess storing function and are cycle limiting in the sense of the clock-cycle algorithm. We concentrate the elements of all pairwise disjoint sets $M_{I}, M_{O}, M_{E}$ and $M_{L}$ to the set of boxes $M_{B}=M_{I} \cup M_{O} \cup M_{E} \cup M_{L}$. On the basis of these sets 
the hardware model can be considered as a directed bipartite graph. Therefore, we introduce the relation $M_{\mathcal{R}} \subseteq\left(M_{B} \times M_{S}\right) \cup\left(M_{S} \times M_{B}\right)$ describing the connections between boxes and signals. Using the sets of successors $\mathcal{N}_{G}^{+}(x)=\{y \mid(x, y) \in \mathcal{R}\}$ and predecessors $\mathcal{N}_{G}^{-}(x)=\{y \mid(y, x) \in \mathcal{R}\}$ for any directed graph $G=(X, \mathcal{R})$ and $x \in X$ we define:

Definition 2.1 Let $M_{I}, M_{O}, M_{E}, M_{L}$, and $M_{S}$ be pairwise disjoint and nonempty sets. $M_{B}$ and $M_{\mathcal{R}}$ are defined as above. $M=\left(M_{I}, M_{O}, M_{E}, M_{L}, M_{S}, M_{\mathcal{R}}\right)$ is called hardware model if the corresponding directed bipartite graph $G(M)=\left(M_{B}, M_{S}, M_{\mathcal{R}}\right)[5,13]$ satisfies the following conditions:

1. $\left\{x \mid x \in M_{B} \cup M_{S} \wedge \mathcal{N}_{G(M)}^{-}(x)=\emptyset\right\}=M_{I}$,

2. $\left\{x \mid x \in M_{B} \cup M_{S} \wedge \mathcal{N}_{G(M)}^{+}(x)=\emptyset\right\}=M_{O}$,

3. any directed cycle in $G(M)$ includes at least one element of $M_{L}$.

$M_{I}$ and $M_{O}$ are the sets of all sources and sinks of $G(M)$, respectively. Condition 3 ensures the absence of directed cycles only including elements of $M_{E} \cup M_{S}$. This corresponds to the exclusion of asynchronous combinational feedbacks.

Due to our parallelization approach, cutting signals of $M_{S}$ during a partitioning of $M$ is only permitted at cycle-boundaries related to the clock-cycle algorithm. Therefore, we are forced to define basic units for partitioning which are known as cones $[12,6,7]$ with respect to an arbitrarily chosen hardware model $M$ :

Definition 2.2 The fan-in cone $\mathrm{co}_{I}(x)$ of an element $x \in M_{O} \cup M_{E} \cup M_{L}$ is recursively defined by:

$$
\begin{aligned}
& \text { 1. } x \in \operatorname{co}_{I}(x), \\
& \text { 2. } y \in M_{E} \wedge \mathcal{N}_{G(M)}^{+}\left(\mathcal{N}_{G(M)}^{+}(y)\right) \cap \operatorname{co}_{I}(x) \neq \emptyset \rightarrow y \in \\
& \operatorname{co}_{I}(x) .
\end{aligned}
$$

The fan-out cone $c_{O}(x)$ of $x \in M_{I} \cup M_{E} \cup M_{L}$ is analogously defined using the sets of predecessors.

Let us take a cone $c o(x)$ as a special fan-in cone the head element $x$ of which satisfies $x \in M_{O} \cup M_{L}$. All the cones form the set $C o(M)$ as the set of basic units for the partitioning of $M$. An example illustrating the introduced sets $M_{I}, M_{O}, M_{E}, M_{L}$ and cones for a simple hardware model is depicted in Fig. 1. The number of cones belonging to $C o(M)$ is $m_{c}=|C o(M)|=\left|M_{L}\right|+\left|M_{O}\right|$. A box $b \in M_{E}$ (logical

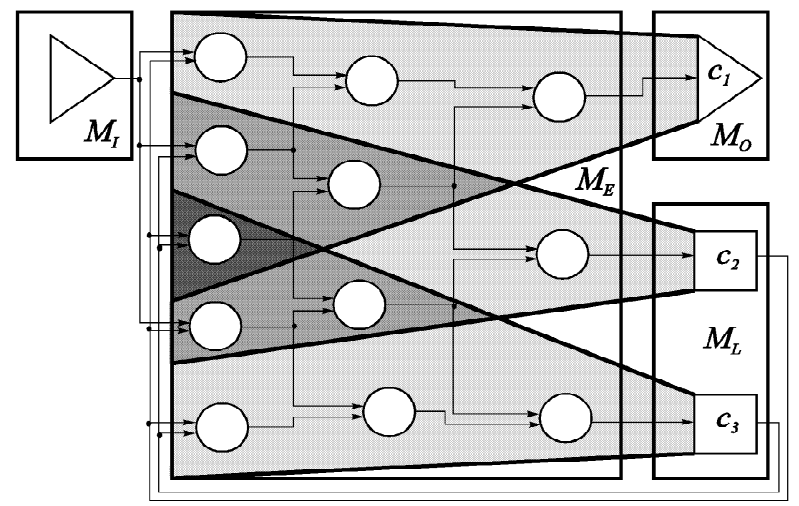

Figure 1 - hardware model with cones (shaded)

element), from which directed paths (with all intermediate boxes being elements of $M_{E}$ ) to the heads of different cones $\hat{c}_{i} \in C o(M), i=1, \ldots, m$ exist, belongs to all of the $m$ different cones $\hat{c}_{i}: b \in \cap_{i=1}^{m} \hat{c}_{i}$. These cones $\hat{c}_{i}$ are called to be overlapping. Considering all cones $c_{i}$ of the model we get:

$$
\sum_{i=1}^{m_{c}}\left|c_{i}\right| \geq\left|\bigcup_{i=1}^{m_{c}} c_{i}\right|=|C o(M)|+\left|M_{E}\right| .
$$

If overlapping cones are distributed to different processors one has to take into account the multiple evaluation of boxes in parallel simulations. ${ }^{2}$ In the following, $\mathcal{C}$ always denotes nonempty subsets of $C o(M)$.

Definition 2.3 1.) The box-related cone overlap degree $u: M_{E} \rightarrow I N$ is defined by $u(b)=$ $|\{c \mid c \in C o(M) \wedge b \in c\}|$, giving the number of cones which contain the box $b \in M_{E}$.

2.) The overlap region ovr $(\mathcal{C})$ of a set of cones $\mathcal{C}$ is the set of boxes belonging to these and only these cones $c \in \mathcal{C}$ :

$$
\operatorname{ovr}(\mathcal{C})=\left(\bigcap_{c \in \mathcal{C}} c\right) \backslash\left(\bigcup_{c^{\prime} \in C o(M) \backslash \mathcal{C}} c^{\prime}\right)
$$

All elements of the set $M_{E} \cup M_{L} \cup M_{O}$ are uniquely distributable into overlap regions $\operatorname{ovr}(\mathcal{C})$. Using $P^{*}=$ $2^{C o(M)} \backslash\{\emptyset\}$ we get:

$$
\sum_{\mathcal{C} \in P^{*}}|\operatorname{ovr}(\mathcal{C})|=|C o(M)|+\left|M_{E}\right|
$$

\footnotetext{
${ }^{2}$ On the other hand, communication between the processors is reduced to communication at the clock-cycle boundaries. Of course, replication in the evaluation of boxes is an additional restricting factor and has to be minimized.
} 
In general, most of the overlap regions $\operatorname{ovr}(\mathcal{C})$ are empty sets. The set of boxes of a cone $c$ is uniquely decomposable into overlap regions $\operatorname{ovr}(\mathcal{C})$ with $c=$ $\bigcup_{\left(\mathcal{C} \in P^{*} \wedge c \in \mathcal{C}\right)} \operatorname{ovr}(\mathcal{C})$ and $|c|=\sum_{\left(\mathcal{C} \in P^{*} \wedge c \in \mathcal{C}\right)}|\operatorname{ovr}(\mathcal{C})|$. The set of all overlap regions $\operatorname{ovr}(\mathcal{C})$ allows the construction of an equivalent weighted overlap hypergraph $G U$ identifying the nodes with cones and the hyperedges with cone sets $\mathcal{C}$ corresponding to nonempty overlap regions $\operatorname{ovr}(\mathcal{C})$ with $|\mathcal{C}|>1[6]$.

Next, we introduce the terms partitioning and partition by means of two nonempty sets $U$ and $V$.

Definition 2.4 1.) A partitioning of $U$ with respect to $V$ is a unique map $\Phi: U \rightarrow V$ assigning each element $u \in U$ to an element $v \in V$.

2.) A partition $\Psi_{\Phi}$ of $U$ related to the partitioning $\Phi: U \rightarrow V$ is given by $\Psi_{\Phi}=\left\{\Phi^{-1}(v) \mid v \in \operatorname{cod} \Phi\right\}$, where $\operatorname{cod} \Phi$ is the range of $\Phi$.

An element $v \in \operatorname{cod} \Phi$ represents the partition component $\Phi^{-1}(v)$ containing all elements $u \in U$ which are mapped onto $v$. Here, we identify $U$ with the set of cones $C o(M)$ and $V$ with the set $\mathcal{B}$ of $m_{b}$ blocks representing processors. If not specified otherwise we consider surjective partitionings.

The task is to find a partitioning $\Phi_{\text {opt }}: C o(M) \rightarrow \mathcal{B}$ for the given sets $C o(M)$ and $\mathcal{B}$, which leads to a significant lower running time $T_{\text {par }}$ for parallel simulation of a clock-cycle in comparison with $T_{\text {seq }}$ in the sequential case. To achieve this goal we consider quality functions taking into account several aspects such as interprocessor communication, workload balance and replication rate influencing $T_{\text {par }}$. Then, for a certain quality function $\Omega$ one has to determine a partitioning $\Phi_{\text {opt }}^{\Omega}$ which optimizes $\Omega$. Currently we consider

$$
\Omega=\max _{B \in \mathcal{B}}(W(B))
$$

as to be minimized where

$$
W(B)=\left|\bigcup_{c \in \Phi^{-1}(B)} c\right| .
$$

$W(B)$ can be interpreted as workload of block $B$ under the assumption of an unique time unit $\tau=1$ for the evaluation of each box. $W(B)$ can be also expressed in terms of overlap regions: $W(B)=$ $\sum_{\left(\mathcal{C} \in P^{*} \wedge \mathcal{C} \cap \Phi^{-1}(B) \neq \emptyset\right)}|\operatorname{ovr}(\mathcal{C})|$. For sequential simulation we have with (2.3) the sequential workload as

$$
W_{\text {seq }}=|C o(M)|+\left|M_{E}\right|
$$

which is equal to the sum of boxes to be evaluated.

\section{Hierarchical Partitioning}

\subsection{Hierarchical Strategy and the Mix- ture of Experts Approach}

In the applications considered here the ratio between $m_{c}$ as the number of cones and $m_{b}$ as the number of blocks may be up to the range of $10^{5}-10^{6}$. Therefore, we focus onto a hierarchical strategy [1] which has been successfully applied to data extensive problems as, for instance, non-linear principle component analysis (PCA) and robotics [10]. To gradually reduce the range of the problem we introduce a general $q$-level partitioning scheme according to Def. 2.4:

Definition 3.1 A q-level partitioning of $U$ with respect to $V$ is defined by $\Phi_{H}: U \rightarrow V$ with $\Phi_{H}=$ $\Phi_{q} \circ \Phi_{q-1} \circ \ldots \circ \Phi_{1}$ where $\Phi_{j}: V_{j} \rightarrow V_{j+1}$ and $V_{1}=U$, $V_{q+1}=V$ and furthermore $\left|V_{1}\right| \geq\left|V_{2}\right| \geq \ldots \geq\left|V_{q+1}\right|$.

Clearly, in general $\Phi_{H}$ is only an approximation of $\Phi_{\text {opt }}$. In our application we use a 2-level scheme $\Phi_{H}=$ $g \circ f$, i.e. $V_{1}=U=C o(M), V_{2}=\mathcal{S}$ and $V_{3}=V=\mathcal{B}$ :

$$
\Phi_{H}: C o(M) \stackrel{f}{\longrightarrow} \mathcal{S} \stackrel{g}{\longrightarrow} \mathcal{B} .
$$

Thereby, $\mathcal{S}$ is a set of elements $S_{l}$, the pre-images $s_{l}=f^{-1}\left(S_{l}\right)$ of which are called super-cones. We remark that super-cones are collections of usual cones. ${ }^{3}$ In contrast to the determination of the cones the realizations of $g$ and $f$ are free. This allows an optimal adaptation. However, often an a priori optimal choice is not possible [5]. To overcome this difficulty we prefer in each level of the hierarchical scheme a strategy introduced in neurodynamics by JORDAN et al. [2] which is called mixture of experts.

For a $q$-level scheme we consider several partitioning algorithms $A_{i}^{j}, i=1 \ldots m_{j}$ corresponding to maps $\Phi_{i}^{j}$ and working in a parallel way in one hierarchical step $j$ representing various partitioning heuristics. The resulting partitions $\Psi_{A_{i}^{j}}$ are compared with respect to a quality measure and the $\beta_{j}$ best of them will form the basis for the algorithms $A_{l}^{j+1}$ of the next level which generate partitions $\Psi_{A_{i}^{j}, A_{l}^{j+1}}$. Thereby, the images of the super-cones of a partition $\Psi_{A_{i}^{j}}$ given by $\Phi_{i}^{j}$ are taken as the new basic units. The final result of a $q-$ level scheme is a partition $\Psi_{A_{i_{1}}^{1}, A_{i_{2}}^{2}, \ldots, A_{i_{q}}^{q}}$ the quality

\footnotetext{
${ }^{3}$ On the other hand, the cones themselves are sets of boxes which are elements of $\mathcal{M}=M_{E} \cup M_{L} \cup M_{O}$. Therefore, we can regard the concentration of these as an initial 'partitioning' $\Phi_{0}: \mathcal{M} \rightarrow C o(M)$. In this way we obtain $\Phi_{H}^{*}=g \circ f \circ \Phi_{0}$ as an extended 2-level scheme. The definition of the cones uniquely determines the map $\Phi_{0}$. Yet, $\Phi_{0}$ is not a partitioning in the sense of Def. 2.4 .
} 
measure of which is the best in the last level. However, as yet this describes only a simple strategy of competing experts.

By introducing superpositions $\tilde{\Psi}$ of a set $\Pi=$ $\left\{\Psi_{1}, \ldots, \Psi_{k}\right\}$ of partitions within a certain level we next extend the competing approach to a mixture one. In this context $\tilde{\Psi}$ plays the role of a generating system, i.e. each super-cone of a partition $\Psi_{i} \in \Pi$ is expressible in terms of super-cones $\tilde{s}_{l} \in \tilde{\Psi}$ :

Definition 3.2 Let $\Pi=\left\{\Psi_{1}, \ldots, \Psi_{k}\right\}$ be a system of partitions of the set $U$. The elements of $\Psi_{i}$ are denoted by $s_{i}^{j}, j=1 \ldots n_{i} . \tilde{\Psi}=\left\{\tilde{s}_{1}, \ldots, \tilde{s}_{m}\right\}$ is called a superposition of $\Pi$ if and only if:

1. $\tilde{\Psi}$ is a partition of $U$

2. $\tilde{\Psi}$ is a generating system for each $\Psi_{i} \in \Pi$, i.e., for each $s_{i}^{j} \in \Psi_{i}\left(i=1 \ldots k, j=1 \ldots n_{i}\right)$ exist $\tilde{s}_{l_{1}}, \ldots, \tilde{s}_{l_{r}} \in \tilde{\Psi}$ such that $s_{i}^{j}=\tilde{s}_{l_{1}} \cup \ldots \cup \tilde{s}_{l_{r}}$.

Def. 2.4 yields $\emptyset \notin \tilde{\Psi}$. The elements of $U$ taken as single sets form a superposition $\tilde{U}$ of $\Pi$. However, we want to have a superposition the granularity of which is rougher than the granularity of $\tilde{U}$, i.e., $|\tilde{U}|>$ $|\tilde{\Psi}|$. Therefore we consider a special construction of superpositions:

Theorem 3.3 Let $\Pi=\left\{\Psi_{1}, \ldots, \Psi_{k}\right\}$ be a system of partitions of the set $U$. The elements of $\Psi_{i}$ are denoted by $s_{i}^{j}, j=1 \ldots n_{i}$. Furthermore, let $\Psi^{*}$ be given as

$$
\Psi^{*}=\left\{s_{j_{1} \ldots j_{k}}^{*} \mid s_{j_{1} \ldots j_{k}}^{*}=\bigcap_{i=1 \ldots k} s_{i}^{j_{i}}\right\} \backslash\{\emptyset\}
$$

with $j_{i}=1 \ldots n_{i}$. Then $\Psi^{*}$ is a superposition of $\Pi$. Furthermore, for all superpositions $\hat{\Psi}$ of $\Pi$ with $\hat{\Psi} \neq$ $\Psi^{*}$ the relation $|\hat{\Psi}|>\left|\Psi^{*}\right|$ is valid, i.e. $\Psi^{*}$ has the maximum granularity.

Proof: The proof of the theorem is shown in the Appendix.

Following the theorem we are able to determine a superposition $\Psi^{*}$ of maximum granularity by $k$-times intersections according to (3.2). Yet, in general we only have $|\tilde{U}| \geq\left|\Psi^{*}\right|$. The structure of $\Psi^{*}$ depends on the properties of the partitions $\Psi_{i} \in \Pi$ which represent the different partitioning heuristics (realized by the corresponding algorithms). Hence, all used strategies influence the superposition, i.e., the expert knowledge of the algorithms is mixed in $\Psi^{*}$. We add a superposition according to Theorem 3.3 as a special partition to the $\beta_{j}$ best of one hierarchical level $j$ so that it may be used in the next level, too.
Returning to our 2-level scheme, the use of a superposition is suitable after the first partitioning level. If we assume that we have various algorithms $A_{i}^{1}$ realizing the maps $f_{i}: C o(M) \rightarrow \mathcal{S}_{i}$ we obtain $\Psi_{i}=f_{i}^{-1}\left(\mathcal{S}_{i}\right)$ according to Def. 2.4. $\mathcal{S}_{i}$ are sets of the mappings of the super-cones determined by the partitions $\Psi_{i}$, respectively. In analogy, we introduce the abstract map $f^{*}: \operatorname{Co}(M) \rightarrow \mathcal{S}^{*}$ where $\mathcal{S}^{*}$ is representing the set of super-cones $s_{l}^{*} \in \Psi^{*}$ and $\Psi^{*}=\left(f^{*}\right)^{-1}\left(\mathcal{S}^{*}\right)$. Then each element of the set system $\Sigma_{f}=\left\{\mathcal{S}_{1}, \ldots, \mathcal{S}_{\beta_{1}}, \mathcal{S}^{*}\right\}$ can be taken as a new system of basic units for partitioning in the second level.

However, the above mixture strategy is a very simple one. In the next section we will improve this strategy using genetic algorithms. Thereby, condition 2 of Def. 3.2 becomes important.

\subsection{Improved Mixture of Experts Ap- proach Using Genetic Algorithms}

In this part we extend the mixture approach introduced in section 3.1 using genetic algorithms (GAs) [1]. In GAs, populations of individuals (parents) produce new individuals (children) in a manner which is inspired by biological evolution and reproduction. The individuals are strings describing a set of parameters which are to be optimized. ${ }^{4}$ For applying GAs to graph partitioning let us consider a partitioning map $\Phi: U \rightarrow V$. Furthermore, one has to optimize $\Phi$ regarding to a certain quality function $\Omega$ (fitness function). In this context an individual $j$ represents a certain partition, determined by a map $\Phi_{j}$. The $i$-th component of the string is associated with the $i$-th element of $U$ containing the mapping goal which is an element of $V$. Several authors have applied GAs to graph partitioning, for instance [8].

However, we will involve this approach into the above described hierarchical strategy. Here we focus onto the 2-level scheme (3.1). In general, GAs may be used in each hierarchical level. Yet, because of the large number of cones in $C o(M)$ the string of an individual representing a partition of $C o(M)$ is often too long for mastering. On the other hand, if applying GAs in the second level of the hierarchical scheme, they require a uniform set of basic elements. To serve this assumption the use of the superposition $\Psi^{*}$ specified in (3.2) of Theorem 3.3 is appropriate because of its property as a generating system. In this context the initial population for the GA is based on the set of all partitions determined in the first level which now are described in terms of the elements of $\mathcal{S}^{*}$. We emphasize again that the several algorithms represent various

\footnotetext{
${ }^{4}$ For a more detailed introduction see for instance [4].
} 
partitioning strategies the best of which a priori is unknown. Still more, in general a merging strategy will improve the result significantly. We can realize such a strategy using the recombination by crossing over in GAs to join different properties of two individuals (partitions) into new ones. The crossing over scheme may be interpreted as a more general exchanging than the simpler one in the algorithm of KERNIGHAN AND LIN [3]. However, we have to take into account a second argument, how much of the old individuals get the chance to be allowed for the competing step (selection) to build the new population. Let us suppose that $\mu$ parent individuals produce $\lambda$ children. Two contrary methods are well known: 1 ) the $\mu$ best of the $\lambda$ children only form the new population with $\mu<\lambda$; 2) all $\mu+\lambda$ individuals are allowed for the selection process. ${ }^{5}$ In the second case the best solution is preserved. Yet, it tends to a stagnation into a local minimum. In the first scheme this property is weakened. On the other hand, good solutions may get lost here. Therefore, we introduce a new so-called $[\mu * \lambda]-$ scheme which balances both strategies: at a time $t$ now $\mu_{t}+\lambda$ individuals have to be taken into consideration with $\mu_{t}=\operatorname{int}\left[\left(\mu-\mu_{\alpha}\right) \cdot \sigma(t)\right]+\mu_{\alpha}$. Thereby, int $[x]$ stands for the integer value of $x$. The function $\sigma(t)$ is of decreasing sigmoid type with $0 \leq \sigma(t) \leq 1$. $\mu_{\alpha}$ describes the final survival probability for the parent individuals. We have $\mu_{0}=\mu$ and $\lim _{t \rightarrow \infty} \mu_{t}=\mu_{\alpha}$.

The whole procedure, which includes the generation of a superposition and following GA, finally leads to the complete scheme of the improved hierarchical mixture strategy depicted in Tab. 3.2.

\subsection{Special Experts}

Our mixture of experts approach is a framework for applying several partitioning algorithms as experts. A survey of algorithms suitable for parallel logic simulation is given in [13]. We distinguish direct and iterative partitioning algorithms which construct a single partition resulting from basic units without building intermediate partitions or require an initial partition which is gradually improved according to a quality function, respectively.

We have developed two new direct algorithms on the basis of cones aiming at balanced workload and minimum replication, the Backward-Cone-Concentration algorithm $(n-B C C)$ and the Minimum-Overlap ConeCluster algorithm $(M O C C)$.

The basic idea of $n-B C C$ consists in iteratively assigning sets of cones to blocks with preferred choice of

\footnotetext{
${ }^{5}$ These correspond to the $[\mu, \lambda]-$ and $[\mu+\lambda]-$ scheme in the notions of RECHENBERG and SCHWEFEL, respectively [9, 11].
}

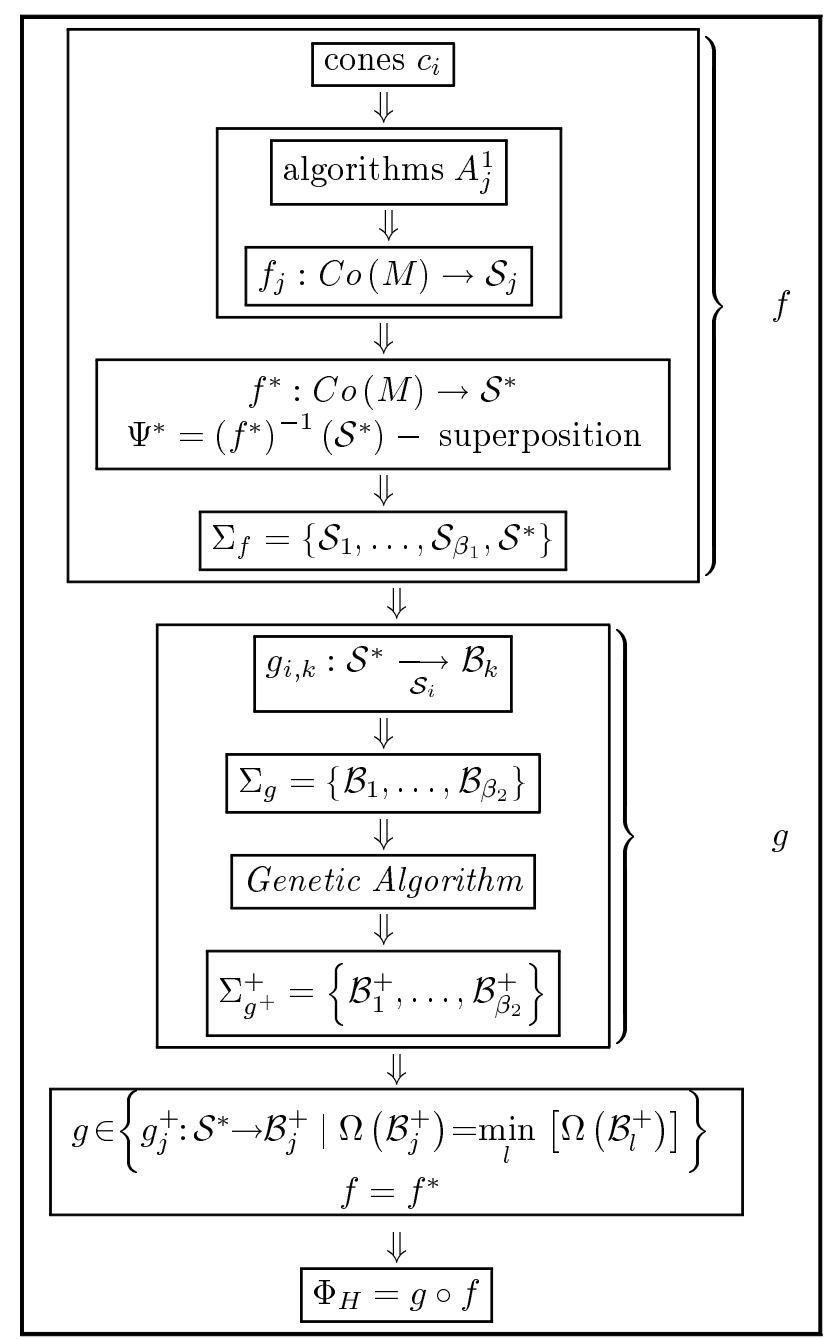

Tab. 3.2. : Scheme of the improved mixture of experts strategy using genetic algorithms

$n$ cones overlapping each other using the box-related cone overlap degree $u$ of Def. 2.3:

1. Fix a value $n^{*}$ with smallest distance to $n$ in the range of $u$; all boxes in $M_{E}$ are assumed to be unmarked.

2. Choose a box $e$ within all unmarked boxes out of $u^{-1}\left(n^{*}\right)$ and search its fan-out cone coo $(e)$ (see Def. 2.2) to find the head elements of the $n^{*}$ cones covering $e$. These $n^{*}$ cones are assigned to a block possessing the lowest number of cones for the moment and all boxes of the selected cones become marked. If there is a remaining unmarked box $e \in u^{-1}\left(n^{*}\right)$, then step 2 is repeated.

3 . If there exists $n^{\prime} \in \operatorname{cod} u$ with $u^{-1}\left(n^{\prime}\right)$ containing an unmarked box, then such a $n^{\prime}$ is taken as 
the new $n^{*}$ and one has to continue with step 2 . Otherwise, the algorithm terminates.

Contrary to $M O C C$ explained below, $n-B C C$ does not explicitly use knowledge concerning the number of boxes in overlap regions or cones. First of all, $n^{-}$ $B C C$ has been designed for application at the first level of our hierarchical strategy.

$M O C C$ successively constructs a partition using the specifics of the weighted overlap hypergraph $G U$ corresponding to the hardware model $M$. With this algorithm the objective is to achieve partitions with blocks containing hypergraph nodes (cones) connected among one another with high-weighted hyperedges:

1. Initially, $m_{b}$ cones of $C o(M)$ are assigned to the $m_{b}$ blocks.

2. Taking block $B_{i} \in \mathcal{B}$ with the lowest number of boxes, we are looking for that overlap region $\operatorname{ovr}\left(\mathcal{C}^{*}\right)$ of $B_{i}$ with $\mathcal{C}^{*} \cap \Phi^{-1}\left(B_{i}\right) \neq$ $\emptyset$ which maximizes the product $\left|\operatorname{ovr}\left(\mathcal{C}^{*}\right)\right|$. $\left|\mathcal{C}^{*} \backslash \bigcup_{j=1 \ldots m_{b}} \Phi^{-1}\left(B_{j}\right)\right|$.

3. Assign the set of these up to now not considered cones $\mathcal{C}^{*} \backslash \bigcup_{j=1 \ldots m_{b}} \Phi^{-1}\left(B_{j}\right)$ concerning the selected overlap region $\operatorname{ovr}\left(\mathcal{C}^{*}\right)$ to the block $B_{i}$.

4. If free cones exist yet, proceed with step 2. Otherwise the partition is complete and the algorithm stops.

$M O C C$ aims at a minimum of multiple evaluation of boxes on different processors keeping a balanced workload corresponding to the resulting partition. If twostage partitioning is necessary the complex structure of $G U$ implies preferably applying $M O C C$ to the second level of the hierarchical partitioning scheme.

\section{Experimental Results - Conclusions}

Finally, we present a special application of the improved mixture of experts strategy (Tab. 3.2) for a specific hardware model $M$ representing a processor structure with $\left|M_{E}\right|=16398$ boxes. ${ }^{6}$

For the initial hierarchical level we use a set of $n$-BCC algorithms $A_{k}^{1}$ with varying parameters $n$ and numbers of super-cones $m_{s}$. The crossing to the second level requires the production of an initial population $\Sigma_{g}$ for the genetic algorithm to be applied. Generally, each $S_{i}$ resulting from the first level allows the production of many individuals $\mathcal{B}_{k}$ in the second level $g_{i . k}: S^{*} \underset{S_{i}}{\longrightarrow} \mathcal{B}_{k}$, using the elements of $S^{*}$ as new basic

\footnotetext{
${ }^{6}$ provided by $I B M$
}

units and keeping such units together in one block of $\mathcal{B}_{k}$ which correspond to one and the same super-cone belonging to $S_{i}$. Here, we restrict the number of created initial individuals $\mathcal{B}_{k}$ to one for each $S_{i}$. For the evaluation of individuals within the genetic algorithm and for choosing the final partition described by $\Phi_{H}$, the quality function $\Omega \underset{(2.4)}{=} \max _{B \in \mathcal{B}}(W(B)$ ) (maximum workload) is taken.

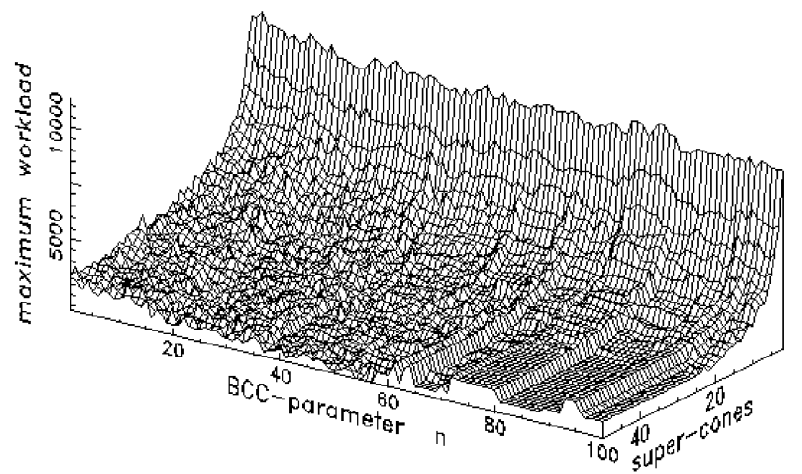

Figure 2 - Quality function $\Omega$ (maximum workload) for partitions resulting from the $n$-BCC for various numbers of super-cones and values of parameters $n$.

In Fig. 2 the quality function $\Omega$, applied to the partitioning results of the first hierarchical level of $n$ $B C C$ for various $m_{s}=2 \ldots 50$ with the parameters $n=1 \ldots 100$, is shown.

In our exemplary application of the hierarchical scheme we investigate 3 cases for application of GAs differing in the initial population which is randomly chosen out of corresponding partitioning results of the first hierarchical level. In all tests the maximum number of blocks is limited to $m_{b}=32$.

First, we build the initial population only from partitions consisting of 32 super-cones, i.e. $m_{s}=32$. The evaluation in time (number of generations) of the fitness according to the best individual (partition) of the population is shown in Fig. 3 (short dashed line). The $\Omega$-value of the fittest individual decreases from 2250 to 1899 with $m_{b}=32$. In the second example all initial partitions are formed over $m_{s}=16$ super-cones. Starting with a best individual of $\Omega_{\text {start }}=2896$ the final solution gives a partition with $\Omega_{\text {end }}=1985$ and $m_{b}=32$ (straight line in Fig. 3). Yet, this is better than the start value in the first case but its final value is not reached. Nevertheless, the difference between $\Omega_{\text {start }}$ and $\Omega_{\text {end }}$ in the second example is more than 900 because there is a high variability in crossing 
from 16 super-cones up to 32 blocks in a partition. Therefore, in the last experiment we merge individuals of varying parameter $n$ of the $n-B C C$ algorithm on the one hand side and individuals with different numbers $m_{s}$ on the other hand in the initial population. In fact, this leads to a better performance, i.e. the final value of the fittest individual now is $\Omega_{\mathrm{end}}=1803$ with $m_{b}=32$ again (long dashed line in Fig. 3 ).

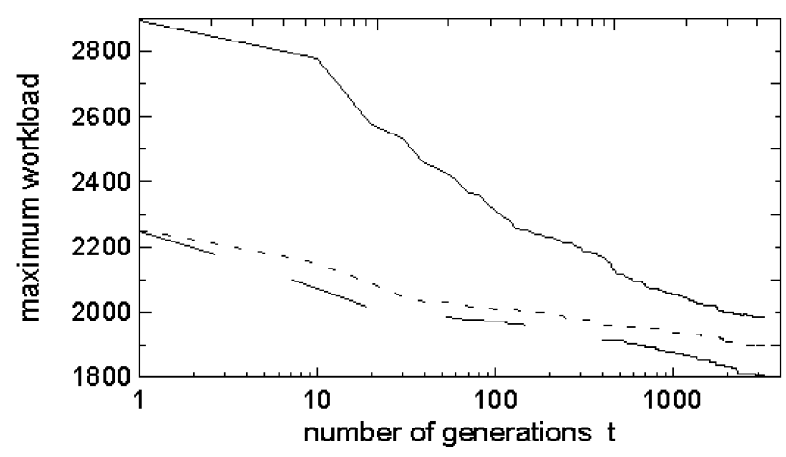

Figure 3 - Maximum workload of the best partition generated by a GA for $m_{s_{1}}=16$ (short dashed), $m_{s_{2}}=32$ (straight line) and for a mixed initial population (long dashed line) with respect to time (number of generations), see text.

These first results show that a mixture of the a priori chosen strategies represented by the various $n-B C C$ instances leads to improved partitions. The successful application of the GAs to the mixture strategy of partitioning algorithms was demonstrated using the idea of superposition of partitions.

\section{Acknowledgement}

This work was supported by DeuTsche Forschungsgemeinschaft (DFG) under the grant SP $487 / 1-2$.

\section{Appendix}

\section{Proof of Theorem 3.3:}

\section{(I) $\Psi^{*}$ is a generating system:}

Let $s_{i}^{j} \in \Psi_{i}$ be arbitrarily chosen. We construct the sets $S_{l}$ with $l \neq i$ according to the following rule: if $s_{i}^{j} \cap s_{l}^{j^{\prime}}=\tilde{s}_{l}^{j^{\prime}}$ with $s_{l}^{j^{\prime}} \in \Psi_{l}$ and $\tilde{s}_{l}^{j^{\prime}} \neq \emptyset$ holds then $\tilde{s}_{l}^{j^{\prime}} \in S_{l}$. Then for each $l$ the relation $\bigcup_{j^{\prime}} \tilde{s}_{l}^{j^{\prime}}=s_{i}^{j}$ is valid. We consider the set $\Xi^{*}=$ $\left\{s_{j_{1}^{\prime} \ldots j_{k}^{\prime}}^{*} \mid s_{j_{1}^{\prime} \ldots j_{k}^{\prime}}^{*}=\underset{\substack{l=1 \ldots k \\ l \neq i}}{\cap} \tilde{s}_{l}^{j_{l}^{\prime}} ; \tilde{s}_{l}^{j_{l}^{\prime}} \in S_{l}\right\} \backslash\{\emptyset\} . \quad \mathrm{Be}-$ cause of the definition of the $\tilde{s}_{l}^{j_{l}^{\prime}}$ as intersections with $s_{i}^{j} \in \Psi_{i}$ we have $s_{j_{1}^{\prime} \ldots j_{k}^{\prime}}^{*} \in \Psi^{*}$ for $s_{j_{1}^{\prime} \ldots j_{k}^{\prime}}^{*} \neq \emptyset$ and, furthermore, $\underset{s_{j_{1}^{\prime} \ldots j_{k}^{\prime}}^{*} \in \Xi^{*}}{\cup} s_{j_{1}^{\prime} \ldots j_{k}^{\prime}}^{\cup} \subseteq s_{i}^{j}$. It still remains to show $\underset{s_{j_{1}^{\prime} \ldots j_{k}^{\prime}}^{*} \in \Xi^{*}}{\cup} s_{j_{1}^{\prime} \ldots j_{k}^{\prime}}^{*} \supseteq s_{i}^{j}$ : We take an arbitrary but fixed $u \in s_{i}^{j}$. For each set $S_{l}$ one and only one $\tilde{s}_{l}^{j_{l}^{*}}$ exists such that $u \in \tilde{s}_{l}^{j_{l}^{*}}$, i.e., $u \in \underset{\substack{l=1 \ldots k \\ l \neq i}}{\cap} \tilde{s}_{l}^{j_{l}^{*}}$ with $\bigcap_{\substack{l=1 \ldots k \\ l \neq i}} \tilde{s}_{l}^{j_{l}^{*}} \in \Xi^{*}$.

(II) $\Psi^{*}$ is a partition of $U$ :

Lemma 5.1 For $s_{i}^{*} \in \Psi^{*}$ and $s_{j}^{*} \in \Psi^{*}$ with $i \neq j$ holds: $s_{i}^{*} \cap s_{j}^{*}=\emptyset$, i.e. the elements of $\Psi^{*}$ are pairwise disjoint.

According to (I) $\Psi^{*}$ is a generating system for the $s_{i}^{j} \in \Psi_{i}$. Furthermore, it is assumed, that all $\Psi_{i}$ are partitions of $U$ themselves. Then one can find for each element $u \in U$ a super-cone $s_{j^{*}}^{*} \in \Psi^{*}$ such that $u \in s_{j^{*}}^{*}$. Lemma 5.1, the proof of which is shown below, ensures that the elements of $\Psi^{*}$ are pairwise disjoint.

(III) $\Psi^{*}$ has the maximum granularity:

Lemma 5.2 Let $\Pi, \hat{\Psi}$ and $\Psi^{*}$ be defined as in Theorem 3.3. Then, for each super-cone $\hat{s} \in \hat{\Psi}$ a supercone $s^{*} \in \Psi^{*}$ exists such that $\hat{s} \subseteq s^{*}$ is valid.

For arbitrary elements $u \in U$ and partitions $\Psi$ of $U$ let $u^{\Psi}$ denote the uniquely determined super-cone $s \in \Psi$ for which $u \in s$ is satisfied. We choose a set $\mathcal{R}$ of elements $u_{1}, u_{2}, \ldots, u_{\left|\Psi^{*}\right|}$ with $u_{j} \in U, j=1 \ldots\left|\Psi^{*}\right|$ in such a way that for the set $\mathcal{R}^{*}=\left\{u_{j}^{\Psi^{*}}|j=1 \ldots| \Psi^{*} \mid\right\}$ the relation $\mathcal{R}^{*}=\Psi^{*}$ holds. Using this construction and Lemma 5.2 we have for each of the considered $u_{j}$ the relation $u_{j}^{\hat{\Psi}} \subseteq u_{j}^{\Psi^{*}}$ with $u_{j}^{\hat{\Psi}} \in \hat{\Psi}$ and $u_{j}^{\Psi^{*}} \in \Psi^{*}$. The sets $u_{j}^{\hat{\Psi}}$ form the set $\stackrel{\wedge}{\mathcal{R}}=\left\{u_{j}^{\hat{\Psi}}|j=1 \ldots| \Psi^{*} \mid\right\}$. Since $\Psi^{*}$ is a partition it follows that $u_{i}^{\Psi^{*}} \cap u_{j}^{\Psi^{*}}=\emptyset$ for $i \neq j$ and therefore we get $u_{i}^{\hat{\Psi}} \cap u_{j}^{\hat{\Psi}}=\emptyset$. This leads to the inequality $|\hat{\Psi}| \geq|\hat{\mathcal{R}}|=\left|\mathcal{R}^{*}\right|$.

Next we derive the corresponding strong inequality. Because of the assumption $\Psi^{*} \neq \hat{\Psi}$ at least one index $j^{\prime}$ exists with $u_{j^{\prime}}^{\hat{\Psi}} \subset u_{j^{\prime}}^{\Psi^{*}}$. Consider an element $\hat{u} \in$ $u_{j^{\prime}}^{\Psi^{*}} \backslash u_{j^{\prime}}^{\hat{\Psi}}$. Using Lemma 5.2 and $\hat{u} \in u_{j^{\prime}}^{\Psi^{*}}$ we get $\hat{u}^{\hat{\Psi}} \subseteq u_{j^{\prime}}^{\Psi^{*} \cdot 7}$ Hence, for each $i=1 \ldots\left|\Psi^{*}\right|$ one has

\footnotetext{
${ }^{7}$ Moreover, we remark that $u_{j^{\prime}}^{\hat{\Psi}} \neq \emptyset$ yields because $\hat{\Psi}$ being a partition and, hence, $\hat{u}^{\hat{\Psi}} \subset u_{j^{\prime}}^{\Psi^{*}}$ is valid.
} 
$u_{i}^{\hat{\Psi}} \cap \hat{u}^{\hat{\Psi}}=\emptyset$. This leads to the inequality

$$
|\hat{\Psi}| \geq\left|\hat{\mathcal{R}} \cup\left\{\hat{u}^{\hat{\Psi}}\right\}\right|=|\hat{\mathcal{R}}|+1>\left|\Psi^{*}\right|
$$

which completes the proof of Theorem 3.3. $\square$

It remains to show that the Lemmata 5.1 and 5.2 hold:

\section{Proof of Lemma 5.1:}

Let $s_{i_{1} \ldots i_{k}}^{*} \in \Psi^{*}$ and $s_{j_{1} \ldots j_{k}}^{*} \in \Psi^{*}$ be given with $\left(i_{1}, \ldots, i_{k}\right) \neq\left(j_{1}, \ldots, j_{k}\right)$. Then an index $l$ exists such that $i_{l} \neq j_{l}$. Further we have $s_{i_{1} \ldots i_{k}}^{*}=s_{1}^{i_{1}} \cap \ldots \cap s_{l}^{i_{l}} \cap$ $\ldots \cap s_{k}^{i_{k}}$ and $s_{j_{1} \ldots j_{k}}^{*}=s_{1}^{j_{1}} \cap \ldots \cap s_{l}^{j_{l}} \cap \ldots \cap s_{k}^{j_{k}}$. Then we obtain for the intersection $s_{i_{1} \ldots i_{k}}^{*} \cap s_{j_{1} \ldots j_{k}}^{*}$ the relation $s_{i_{1} \ldots i_{k}}^{*} \cap s_{j_{1} \ldots j_{k}}^{*}=s_{1}^{i_{1}} \cap s_{1}^{j_{1}} \cap \ldots \cap s_{l}^{i_{l}} \cap s_{l}^{j_{l}} \cap \ldots \cap s_{k}^{j_{k}} \cap s_{k}^{j_{k}}$. The definition of $\Psi^{*}$ in (3.2) yields $s_{l}^{i_{l}} \in \Psi_{l}$ and $s_{l}^{j_{l}} \in \Psi_{l}$. Because $\Psi_{l}$ is a partition of $U, s_{l}^{i_{l}} \cap s_{l}^{j_{l}}=\emptyset$ is valid. $\square$

\section{Proof of Lemma 5.2:}

For the proof of the lemma we show that the following assumption leads to a contradiction:

assumption: There exists a super-cone $\hat{s} \in \hat{\Psi}$ such that it could not be found a super-cone $s^{*} \in \Psi^{*}$ with $\hat{s} \subseteq s^{*}$.

Consider a super-cone $\hat{s} \in \hat{\Psi}$ satisfying the above assumption. Because $\Psi^{*}$ is a partition $|\hat{s}| \geq 2$ follows. In particular, there exist 2 elements $u_{1}, u_{2} \in \hat{s}$ with 1) $u_{1} \in s_{1}^{*}$ and $s_{1}^{*} \in \Psi^{*}$ and 2) $u_{2} \in s_{2}^{*}$ and $s_{2}^{*} \in$ $\Psi^{*}$ such that $s_{1}^{*} \neq s_{2}^{*}$ is valid. Let these be given as $s_{1}^{*}=\bigcap_{i=1 \ldots k} s_{i}^{j_{i}}=s_{j_{1} \ldots j_{k}}^{*}$ and $s_{2}^{*}=\bigcap_{i=1 \ldots k} s_{i}^{h_{i}}=s_{h_{1} \ldots h_{k}}^{*}$. Since $s_{1}^{*} \neq s_{2}^{*}$ one can find an index $l \in\{1, \ldots, k\}$ for which $j_{l} \neq h_{l}$ holds. For the corresponding supercones $s_{l}^{j_{l}}$ and $s_{l}^{h_{l}}$ we have: $s_{l}^{j_{l}}, s_{l}^{h_{l}} \in \Psi_{l}$ and, hence, we get

$$
s_{l}^{j_{l}} \cap s_{l}^{h_{l}}=\emptyset .
$$

Furthermore, $\hat{\Psi}$ is a superposition of $\Pi$, i.e., one can describe the super-cone $s_{l}^{j_{l}}$ in terms of the elements of $\hat{\Psi}$. First, we remark that the relations $u_{1} \in \hat{s} \cap s_{1}^{*}$, $u_{2} \in \hat{s} \cap s_{2}^{*}, s_{1}^{*} \subseteq s_{l}^{j_{l}}$ and $s_{2}^{*} \subseteq s_{l}^{h_{l}}$ lead to

$$
\hat{s} \cap s_{l}^{j_{l}} \neq \emptyset \quad \text { and } \quad \hat{s} \cap s_{l}^{h_{l}} \neq \emptyset \text {. }
$$

Hence, there exists a decomposition of the super-cone $s_{l}^{j_{l}}$ into super-cones $\hat{s}_{r} \in \hat{\Psi}$ :

$$
s_{l}^{j_{l}}=\bigcup_{r=1 \ldots t} \hat{s}_{r}
$$

and we get $\hat{s} \cap s_{l}^{j_{l}} \underset{\text { Eq.(5.4) }}{\overline{\bar{s}}} \hat{\mathrm{s}} \cap\left(\underset{r=1 \ldots t}{\cup} \hat{s}_{r}\right)=\underset{r=1 \ldots t}{\bigcup}$ $\left(\hat{s} \cap \hat{s}_{r}\right) \underset{\text { Eq. (5.3) }}{\neq} \emptyset$. Then an index $r^{*}$ exists with $\hat{s}=\hat{s}_{r^{*}}$, i.e.

$$
s_{l}^{j_{l}}=\hat{s}_{1} \cup \ldots \cup \hat{s} \cup \ldots \cup \hat{s}_{t} .
$$

On the other hand, from (5.3) it follows that one can find an element $\tilde{u} \in \hat{s} \cap s_{l}^{h_{l}}$ which implies $\tilde{u} \in \hat{s}$ and therefore with (5.5) $\tilde{u} \in s_{l}^{j_{l}}$. Yet, this is a contradiction to (5.2) and the lemma is shown. $\square$

\section{References}

[1] K. Hering, R. Haupt, and T. Villmann. An Improved Mixture of Experts Approach for Model Partitioning in VLSI-Design Using Genetic Algorithms. Technical Report 14, University of Leipzig / Inst. of Informatics, Germany, 1995.

[2] M. I. Jordan and R. A. Jacobs. Hierarchical Mixture of Experts and the EM Algorithm. In P. Morasso, editor, Proc. ICANN'94, pages 479486. Springer, 1994.

[3] B. W. Kernighan and S. Lin. An efficient heuristic procedure for partitioning graphs. Bell Systems Technical Journal, 49(2):291-307, 1970.

[4] J. R. Koza. Genetic Programming. MIT Press, 1992.

[5] T. Lengauer. Combinatorial Algorithms for Integrated Circuit Layout. Teubner-Verlag Stuttgart and JOHN WILEY \& SONS, 1990.

[6] N. Manjikian. High performance parallel logic simulation on a network of workstations. TechReport T-220, CCNG, University of Waterloo, 1992.

[7] R. B. Mueller-Thuns, D. G. Saab, R. F. Damiano, and J. A. Abraham. VLSI Logic and Fault Simulation on General Purpose Parallel Computers. IEEE Trans, on Computer-Aided Design of Integrated Circuits and Systems, 12:446-460, 1993.

[8] H. Mühlenbein, M. Gorges-Schleuter, and O. Krämer. Evolution Algorithm in Combinatorial Optimization. Parallel Computing, (7):6588, 1988.

[9] I. Rechenberg. Evolutionsstrategie - Optimierung technischer Systeme nach Prinzipien der biologischen Information. Fromman Verlag Freiburg (Germany), 1973.

[10] H. Ritter, T. Martinetz, and K. Schulten. Neural Computation and Self-Organizing Maps: An Introduction. Addison-Wesley, Reading, MA, 1992.

[11] H.-P. Schwefel. Numerical Optimization of Computer Models. Wiley and Sons, 1981. 
[12] S. P. Smith, B. Underwood, and M. R. Mercer. An analysis of several approaches to circuit partitioning for parallel logic simulation. In Proceedings IEEE International Conference on Computer Design (ICCD), pages 664-667, 1987.

[13] C. Sporrer. Verfahren zur Schaltungspartitionierung für die parallele Logiksimulation. Verlag Shaker Aachen, 1995.

[14] W. G. Spruth. The Design of a Microprocessor. Springer Berlin, Heidelberg, 1989. 